\title{
Commentary on Pain Behaviors in Dementia: Letter to the Editor with Reference to the Article by Morrison et al. (2020)
}

\author{
Mustafa Atee ${ }^{a, b}$ Thomas Morris ${ }^{c}$ Stephen Macfarlane ${ }^{c, d}$ Jeffery D. Hughes ${ }^{b}$ \\ Colm Cunningham ${ }^{c, e}$
}

\begin{abstract}
${ }^{a}$ The Dementia Centre, HammondCare, Wembley, WA, Australia; ${ }^{b}$ School of Pharmacy and Biomedical Sciences, Faculty of Health Sciences, Curtin University, Bentley, WA, Australia; ${ }^{\mathrm{C}}$ The Dementia Centre, HammondCare,

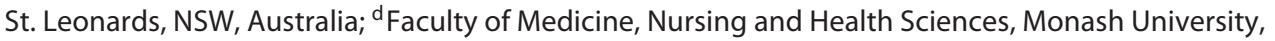

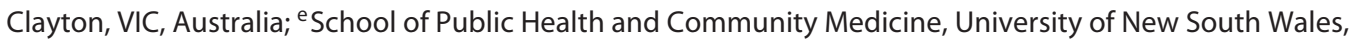
Sydney, NSW, Australia
\end{abstract}

To the Editor,

We read with keen interest the recent study by Morrison et al. [1], entitled "Differences in Staff-Assessed Pain Behaviors among Newly Admitted Nursing Home Residents by Level of Cognitive Impairment," published in the journal Dementia and Geriatric Cognitive Disorders. In the period 2010-2016, the research examines differences in the prevalence of pain behaviors among US nursing home residents $(n=1,036,806)$ with varying levels of cognitive impairment (CI) [1].

In this study, there are a number of interesting findings: 1. The association between the level of CI and the frequency of documentation of pain behaviors, established using modified Poisson models. Of particular note, newly admitted residents with moderate-to-severe CI were less likely to have their pain documented/ assessed by aged care staff compared to those with no or mild CI [1]. This may explain the undertreatment of pain in the residential aged care setting, particularly in those living with later stages of dementia [2]. We know that the correct recognition of pain in residents with moderate-severe CI is a key factor to identify those at greatest risk of undertreatment and to deliver optimal pain management.
(C) 2021 The Author(s)

Published by S. Karger AG, Basel

This article is licensed under the Creative Commons AttributionNonCommercial-NoDerivatives 4.0 International License (CC BYNC-ND) (http://www.karger.com/Services/OpenAccessLicense) Usage and distribution for commercial purposes as well as any distribution of modified material requires written permission.
2. Regardless of CI, residents with no vocal complaints ( $n$ $=144,061$ ) had more frequent documentation of nonverbal pain behaviors (such as, facial expressions and body movements), suggesting that this population may have more reliance on these behaviors to convey the experience of pain. This may also be an evolutionary and compensatory mechanism for partial or complete loss of verbal communication of pain [3].

3. Compared to those with no or mild CI, facial expressions of pain are more commonly documented for residents with moderate-to-severe CI, independently of the effect of covariates, such as depression and anxiety [1]. This finding is not surprising and aligns well with previous literature [4-6]. Thus, the need to use validated facial cues in pain assessment tools [e.g., Pain Assessment Checklist for Seniors with Limited Ability to Communicate (PACSLAC-II), PainChek ${ }^{\circledR}$ (formerly known as the electronic Pain Assessment Tool "ePAT")] for this population [7-9]. Further, the authors rightly point out the importance of using novel and automated methods in capturing facial expressions of pain in an attempt to improve pain assessment in this population $[8,10]$. These methods have proven to be effective in recognizing pain with strong psycho- 
metric and clinimetric properties in people living with dementia [8, 11-13].

In order to advance this area of research, a rich realworld data source like the one reported by Morrison et al. is crucial for our understanding of pain behaviors in cognitively impaired individuals. Given the first objective of this study, i.e. “...to provide a comprehensive description of nonverbal pain behaviors...," it would be reasonable to report the frequency of specific nonverbal pain behaviors in aged care residents with moderate-severe CI, including those living with dementia. However, this is not possible given the limitations of the Minimum Data Set 3.0 as it is a health screening test that does not collect such information and is only confined to an abstract list of "indicators of pain or possible pain in the last 5 days." This additional information might have been better addressed if the collected data were using more specific and itemized descriptors - such as coarse or fine-grained facial expressions of pain (e.g., grimace or brow lowering, respectively). Further, when evaluating the impact of pain on the care needs, collecting more granular data would add a much greater clinical value over generalized behavior descriptors. This is particularly useful if the description of pain behaviors can be reasonably attributed to individual-level data. Another clinically useful approach is the utilization of "big" data in identifying pain (assessment) patterns (e.g., diurnal vs. nocturnal or rest vs. movement pain scores). Using a combination of various technologies such as artificial intelligence, smart automation, internet of things and cloud computing makes capturing real-world data on a large scale a tangible possibility. The above (i.e., data-driven and technology-based) approaches would improve the epidemiological evidence on pain behaviors in people with CI or dementia, both quantitatively and qualitatively.

Finally, we commend the data reported by Morrison et al. in their article and appreciate any additional clinical or demographic information (e.g., pain behaviors by ethnicity; orofacial pain data) related to this important topic.

\section{References}

Assessing Pain Behaviors in Dementia

\section{Acknowledgments}

The authors wish to thank Ms. Marie Alford and The Dementia Centre, HammondCare, for their support with the paper.

\section{Statement of Ethics}

Ethics approval was not required as the submitted manuscript is a letter to the editor.

\section{Conflict of Interest Statement}

Mustafa Atee and JeffHughes are co-inventors of the PainChek ${ }^{\circledR}$ instrument, which is marketed by PainChek Ltd. (ASX: PCK). Both are also shareholders of PainChek Ltd. Mustafa Atee previously held the position of a Senior Research Scientist (October 2018 to May 2020) at PainChek Ltd. and is currently serving the position of Research and Practice Lead at The Dementia Centre, HammondCare. He is also a PhD Candidate at School of Pharmacy and Biomedical Sciences, Curtin University. Jeff Hughes currently holds the position of Chief Scientific Officer at PainChek Ltd., while serving as a Professor at School of Pharmacy and Biomedical Sciences, Curtin University. The co-inventors had a granted patent titled "A pain assessment method and system; PCT/ AU2015/000501" in Australia, China, Japan and the USA, which was assigned to PainChek Ltd. The remaining authors have no further conflicts of interests.

\section{Funding Sources}

This research did not receive any specific grant from funding agencies in the public, commercial, or not-for-profit sectors.

\section{Author Contributions}

M.A. wrote the initial draft of the letter and conducted the literature review. The remaining authors reviewed and contributed to the draft. All authors approved the final version of the letter.
1 Morrison RA, Jesdale BM, Dubé CE, Nunes AP, Bova CA, Liu SH, et al. Differences in staff-assessed pain behaviors among newly admitted nursing home residents by level of cognitive impairment. Dement Geriatr Cogn Disord. 2020;49(3):243-51.

2 Plooij B, van der Spek K, Scherder EJ. Pain medication and global cognitive functioning in dementia patients with painful conditions. Drugs Aging. 2012 May;29(5):377-84.
3 Williams AC. Facial expression of pain: an evolutionary account. Behav Brain Sci. 2002 Aug;25(4):439-55.

4 AGS Panel on Persistent Pain in Older Persons. The management of persistent pain in older persons. J Am Geriatr Soc. 2002 Jun; 50(6 Suppl):S205-24. 
5 Oosterman JM, Zwakhalen S, Sampson EL, Kunz M. The use of facial expressions for pain assessment purposes in dementia: a narrative review. Neurodegener Dis Manag. 2016 Apr; 6(2):119-31.

6 Herr K, Zwakhalen S, Swafford K. Observation of pain in dementia. Curr Alzheimer Res. 2017;14(5):486-500.

7 Chan S, Hadjistavropoulos T, Williams J, Lints-Martindale A. Evidence-based development and initial validation of the pain assessment checklist for seniors with limited ability to communicate-II (PACSLAC-II). Clin J Pain. 2014 Sep;30(9):816-24.
8 Atee M, Hoti K, Hughes JD. Psychometric evaluation of the electronic Pain Assessment Tool (ePAT): an innovative instrument for individuals with moderate to severe dementia. Dement Geriatr Cogn Disord. 2017;44(56):256-67.

9 Atee M, Hoti K, Hughes JD. A technical note on the PainChekTM system: a web portal and mobile medical device for assessing pain in people with dementia. Front Aging Neurosci. 2018 Jun;10:117.

10 Atee M, Hoti K, Parsons R, Hughes JD. A novel pain assessment tool incorporating automated facial analysis: interrater reliability in advanced dementia. Clin Interv Aging. 2018 Jul;13:1245-58.
11 Atee M, Morris T, Macfarlane S, Cunningham C. Pain in dementia: prevalence and association with neuropsychiatric behaviors. J Pain Symptom Manage. DOI: 10.1016/j. jpainsymman.2020.10.011. Epub 2020 Oct 20.

12 Atee M, Hoti K, Parsons R, Hughes JD. Pain assessment in dementia: evaluation of a pointof-care technological solution. J Alzheimers Dis. 2017;60(1):137-50.

13 Hoti K, Atee M, Hughes JD. Clinimetric properties of the electronic Pain Assessment Tool (ePAT) for aged-care residents with moderate to severe dementia. J Pain Res. 2018 Jun;11: 1037-44. 